\title{
First-principles investigations of the magnetic phase diagram of $\mathrm{Gd}_{1-x} \mathrm{Ca}_{x} \mathrm{MnO}_{3}$
}

\author{
Hichem Ben Hamed, ${ }^{1, *}$ Martin Hoffmann, ${ }^{2},{ }^{\dagger}$ Waheed A. Adeagbo, ${ }^{1}$ Arthur Ernst, ${ }^{2,3}$ \\ Wolfram Hergert, ${ }^{1}$ Teemu Hynninen, ${ }^{4}$ Kalevi Kokko, ${ }^{5,6}$ and Petriina Paturi ${ }^{4}$ \\ ${ }^{1}$ Institute of Physics, Martin Luther University Halle-Wittenberg, Von-Seckendorff-Platz 1, 06120 Halle, Germany \\ ${ }^{2}$ Institute for Theoretical Physics, Johannes Kepler University Linz, Altenberger Straße 69, 4040 Linz, Austria \\ ${ }^{3}$ Max Planck Institute of Microstructure Physics, Weinberg 2, 06120 Halle, Germany \\ ${ }^{4}$ Wihuri Physical Laboratory, Department of Physics and Astronomy, University of Turku, FI-20014 Turku, Finland \\ ${ }^{5}$ Department of Physics and Astronomy, University of Turku, FIN-20014 Turku, Finland \\ ${ }^{6}$ Turku University Centre for Materials and Surfaces (MatSurf), Turku, Finland
}

(Dated: April 9, 2019)

\begin{abstract}
We studied for the first time the magnetic phase diagram of the rare-earth manganites series $\mathrm{Gd}_{1-x} \mathrm{Ca}_{x} \mathrm{MnO}_{3}$ (GCMO) over the full concentration range based on density functional theory. GCMO has been shown to form solid solutions. We take into account this disordered character by adapting special quasi random structures at different concentration steps. The magnetic phase diagram is mainly described by means of the magnetic exchange interactions between the Mn sites and Monte Carlo simulations were performed to estimate the corresponding transition temperatures. They agree very well with recent experiments. The hole doped region $x<0.5$ shows a strong ferromagnetic ground state, which competes with A-type antiferromagnetism at higher $\mathrm{Ca}$ concentrations $x>0.6$.
\end{abstract}

\section{INTRODUCTION}

Transition metal oxides are of current interest and constitute one class of promising materials to spawn diverse semiconductor devices [1]. They exhibit a wide range of exotic properties, owing mainly to the partly filled $d$ shell [2]. The hybridization between oxygen $p$ states and the strongly correlated $3 d$ states induce intriguing spin, charge and orbital ordering. These properties are stimulated by the close interplay of structural, electronic and magnetic degrees of freedom. The discovery of the colossal magneto resistance (CMR) effect [3, 4] has triggered an intensive study of the series of rare-earth manganese oxides with general formula $R \mathrm{MnO}_{3}$ (with variable $R=\mathrm{La}, \mathrm{Ce}, \ldots)$.

The $R \mathrm{MnO}_{3}$ series consists of insulating perovskites, which show a multitude of antiferromagnetic (AFM) structures earlier studied by Kimura et al. [5]. The observed A-type AFM (A-AFM) ground state was associated with the tilting of the $\mathrm{MnO}_{6}$ octahedron, known as $\mathrm{GdFeO}_{3}$-type distortion. This kind of distortion becomes even more pronounced for smaller ionic radius of the rare-earth ions $\left(r_{R}\right)$.

Due to the perovskite structure of $R \mathrm{MnO}_{3}$, the resulting crystal-field breaks the degeneracy of the $\mathrm{Mn}^{3+} d$ orbitals. Thus, they split into two degenerated orbitals $\left(e_{\mathrm{g}}\right)$ and three degenerated orbitals $\left(t_{2 \mathrm{~g}}\right)$. The strong Hund's coupling favors the parallel alignment of the four electrons in the majority spin channel. The cooperative Jahn-Teller distortions lift in addition the double degeneracy of the $e_{\mathrm{g}}$ orbitals, while the $t_{2 \mathrm{~g}}$ orbitals become localized. The electrons occupying the $e_{\mathrm{g}}$ orbitals can

\footnotetext{
* hichem.ben-hamed@physik.uni-halle.de

$\dagger$ martin.hoffmann@jku.at
}

in turn hop between the Mn sites through the $p$ orbitals of oxygen. This mechanism is known as the double exchange interaction mechanism and was earlier introduced in the works of Zener [6] and Anderson [7].

Recently, a special focus on $R \mathrm{MnO}_{3}$ was raised because additional features can be accessed by modulating the electrical charge carrier density. That can be realized, e.g., with applying an electrostatic field [8] or chemical doping by introducing alkaline earth elements (abbreviated as $A$ ) at the $R$ site.

The incorporation of alkaline earth elements is the method we want to focus on in this work because the $R \mathrm{MnO}_{3}$ perovskite structure is very robust against adding other ions. It is already widely used since the early works of Wollan and Koehler [9] and Goodenough [10]. Several material systems were already investigated and show full miscibility between the $R$ and $A$ elements, e.g., the $\mathrm{La}_{1-x} \mathrm{Ca}_{x} \mathrm{MnO}_{3}$ series (LCMO) [9, 10], or the $\mathrm{Pr}_{1-x} \mathrm{Ca}_{x} \mathrm{MnO}_{3}$ series (PCMO) [11]. In these solid solutions, the substitution of $R$ ions by $A$ ions causes the Mn $e_{\mathrm{g}}$ electrons to hop to the neighboring ions - a four-valent Mn ion appears. Consequently, two types of manganese emerge in the cell, namely, $\mathrm{Mn}^{3+}$ and $\mathrm{Mn}^{4+}$, and such systems are called mixed valence manganites.

A prominent member of the $R \mathrm{MnO}_{3}$ series is $\mathrm{GdMnO}_{3}$. The main reason is its location in the magnetoelectric phase diagram of the $R \mathrm{MnO}_{3}$ compounds as a function of $r_{R}$ : in close vicinity of the collinear A-type AFM phase but also close to a ferroelectric state $[13,14]$. Hence, the phases could be manipulated rather easily by external means. Kimura et al. [13] found, for instance, that a magnetic field of about $1 \mathrm{~T}$ is sufficient to produce ferroelectricity. On the other hand, $\mathrm{GdMnO}_{3}$ could be also an important candidate for future magneto-optic devices because of its strong magneto-dielectric coupling [15].

Beiranvand et al. [16] studied the magnetic phase diagram of the $\mathrm{Gd}_{1-x} \mathrm{Ca}_{x} \mathrm{MnO}_{3}$ series (GCMO) using mag- 

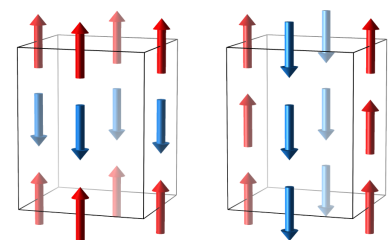

A-AFM

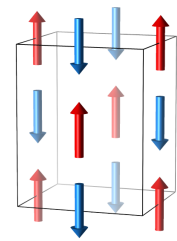

G-AFM
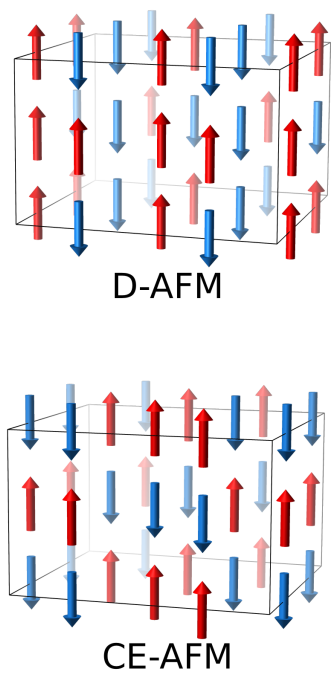
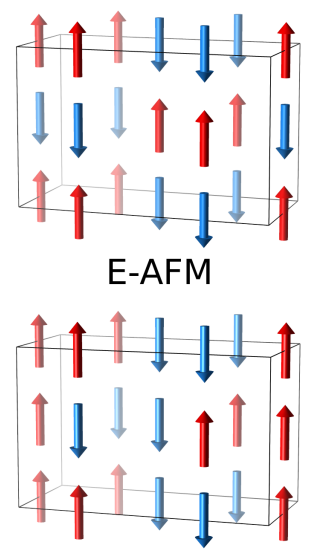

H-AFM
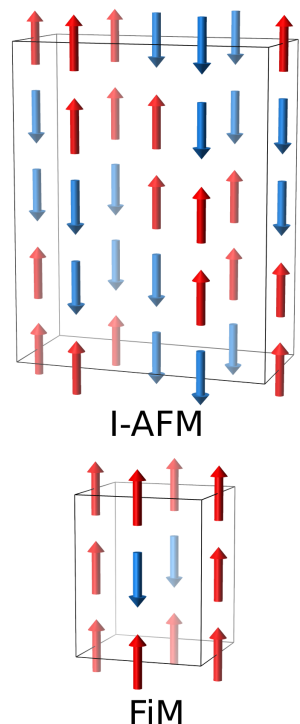

FIG. 1. The different magnetic ground state structures which were suggested in [9] and were taken into account in this work. Here, only the magnetic moments at the Mn sites are represented as arrows - red for the majority and blue for the minority spin direction. Different numbers of repeated Pbnm unit cells (see Fig. 2) are needed to depict the antiferromagnetic (AFM) structures. The opacity of the arrows has no particular meaning but only serves the perspective view. Structural figures were prepared with VESTA [12].

netoresistive measurements of magnetoresistivity in order to understand basic properties of this system. They reported a rich and complicated magnetic phase diagram where the CMR effect showed up at doping concentrations between $x=0.8$ and $x=0.9$. The ferromagnetic insulating phase (FMI) in the region $x<0.5$ transforms for $x>0.5$ to an AFMI phase. A charge ordering $(\mathrm{CO})$ state is found in the concentration range $0.5 \leq x \leq 0.7$ with a maximal $\mathrm{CO}$ transition temperature of about $270 \mathrm{~K}$ at $x=0.5$. Unlike many doped manganites, there is no indication of a metal-insulator transition below the experimental limit of $9 \mathrm{~T}$.

Nevertheless, the underlying microscopic mechanisms are not yet fully understood: The entire character of the magnetic phases is unknown, because Gd and related compounds cannot be easily investigated by means of Neutron diffraction. In fact, $\mathrm{Gd}$ has shown to be the strongest neutron-absorbent among all natural elements [17].

At this point, our theoretical study allows to identify the magnetic ground state from total energy calculations for various potential magnetic phases (see Fig. 1).

We reexamine at first the two undoped systems $\mathrm{GdMnO}_{3}$ (GMO) and $\mathrm{CaMnO}_{3}(\mathrm{CMO})$ as a benchmark for our density functional (DFT) calculations. But when we consider the different concentrations of the solid solution GCMO, the disorder complicates the supercell calculations, necessary to cover all magnetic structures given in Fig. 1.

On the one hand, disorder could be taken into account by an effective medium theory - namely the coherent potential approximation (CPA) in the framework of the Korringa-Kohn-Rostocker Green's function (KKRGF) method [18, 19]. Another elegant way to model disordered systems pioneered by Zunger et al. [20] is the concept of special quasi random structures (SQS) for the rare-earth site mixed with Ca. We decided to use the latter approach because it allows lattice relaxations and could also cover to some degree short-range order effects, which should be compared with experimental results later.

The magnetic properties are discussed in terms of magnetic exchange interactions between the Mn sites. They are then used in a classical Heisenberg model in order to determine the critical magnetic transition temperatures, which agree very well with the experimental results [16]. As the main result, we obtain the type of magnetic ground states, which could not be accessed directly from the magnetoresistance experiments in [16].

\section{UNDOPED MANGANITES}

A lot of work has already been carried out on the theoretical description of both endpoint compounds in the GCMO series. We refer the reader for more details to [21-26] for $\mathrm{GdMnO}_{3}$ and [27-32] for $\mathrm{CaMnO}_{3}$. We aim at the beginning to validate the structural, electronic and magnetic properties against the previous theoretical and experimental results as a benchmark for the following discussion of the phase diagram in section III.

Our density functional theory (DFT) calculations were carried out with the projector augmented-wave method $[33,34]$ as implemented in the Vienna ab initio simulation package (VASP) $[35,36]$. For the treatment of the exchange correlation potential, we compared four common functionals: Perdew-Burke-Ernzerhof (PBE) [37], its revised version for solids (PBEsol) [37], Perdew-Wang (PW91) [38], and Perdew-Zunger (PZ) [39]. An isotropic screened on-site Coulomb interaction [40] - the Hubbard $U$ correction - was added to all aforementioned function- 
als. The choice for the exchange correlation functional and $U$ was made based on the best compromise between the three most important properties: the electronic band gap, the magnetic moment, and primarily the stability of the magnetic order. From those properties, we considered $\mathrm{PBE}+U$ with an $U$ applied on the Mn $3 d$ orbitals with $U_{\mathrm{Mn}}=2 \mathrm{eV}$ as the best choice, while the Gd $f$-electrons are treated as frozen in the core region (motivated by the magnetic properties, see section IIB). A thorough discussion and comparison is given in the supplemental material [41].

\section{A. Lattice and electronic structure}

Both compounds crystallize in the orthorhombic structure with the Pbnm symmetry of the space group 62 including 20 sites (Fig. 2) [42, 43]. The Gd or Ca atoms occupy the $4 c$ Wyckoff position $\left(x_{R}, y_{R}, 1 / 4\right)$, while the Mn atoms are at the $4 b$ Wyckoff position $(1 / 2,0,0)$. The oxygen atoms are located at two different sites and are denoted as $\mathrm{O}_{1}$ for $4 c\left(x_{\mathrm{O}_{1}}, y_{\mathrm{O}_{1}}, 1 / 4\right)$ and $\mathrm{O}_{2}$ for $8 d$ $\left(x_{\mathrm{O}_{2}}, y_{\mathrm{O}_{2}}, z_{\mathrm{O}_{2}}\right)$ (see Table I). The first type of oxygen ions $\left(\mathrm{O}_{1}\right)$ forms bonds with the $\mathrm{Mn}$ in $z$ direction, while the second type $\left(\mathrm{O}_{2}\right)$ is bonded to $\mathrm{Mn}$ ions in the $(x y)$ plane (see Fig. 2).

The orthorhombic structure remains also the lattice structure for the whole $\mathrm{Gd}_{1-x} \mathrm{Ca}_{x} \mathrm{MnO}_{3}$ series for all $\mathrm{Ca}$ concentrations $x$ as the experimental measurements by Beiranvand et al. [16] confirm. We adapt therefore the Pbnm symmetry in all following calculations, either as the primitive cell with 20 sites or as a supercell repeating the Pbnm cell $2 \times 2 \times 2$ times - in total 160 sites. The latter has to be adapted as stated already above in the introduction because we have to take into account all potential magnetic spin orientations (Fig. 1), as well as the disordered character of a solid solution (see section III). As a consequence, the numerical calculation of the volume relaxation and the relaxation of the internal coordinates is too time consuming, because of the large number of sites in the supercell. Hence, we fix the lattice constants of the Pbmn cell to the measured values $[16,44]$, but the internal coordinates could not be accessed by the latter references, and had to be obtained by numerical relaxations. In particular for $x \neq 0$ or 1 in GCMO, the experimental internal parameters are not yet available. For that reason, we validate our numerical results for GMO and CMO against the experimental data in [42, 43] (Table I). The lattice parameters [16, 44] are used and the internal coordinates are allowed to relax [41]. This is referred as $V_{0}$ calculation scheme in Table I.

For GMO and CMO, the internal coordinates vary only slightly from the experimentally obtained atomic positions (Table I). The resulting $\mathrm{Mn}-\mathrm{O}$ bond lengths are in good agreement with those in [42, 43]. For comparison, we calculated also the full volume relaxation. The results are marked as $V_{\text {rlx }}$ calculation scheme in Table I. The GMO volume is slightly overestimated by about
TABLE I. Experimental and calculated structural properties of GMO and CMO. The lattice constants $(a, b$, and $c)$ and the bond lengths $d_{z}, d_{x 1}$ and $d_{x 2}$ are given in $\AA$. The latter correspond to $(\mathrm{Mn}-\mathrm{O})_{z},(\mathrm{Mn}-\mathrm{O})_{x 1}$ and $(\mathrm{Mn}-\mathrm{O})_{x 2}$, respectively (see Fig. 2). The Wyckoff positions $(x, y, z)$ are given in units of the lattice vectors (see text). The Baur's distortion index $B_{\mathrm{D}}$ is dimensionless. The angle variance $\sigma^{2}$ is in (degree) ${ }^{2}$. Note that the $V_{0}$ calculation scheme uses experimental lattice constants [44] and the ferromagnetic Mn spin ordering (section II B).

\begin{tabular}{|c|c|c|c|c|c|c|}
\hline & \multicolumn{3}{|c|}{$\mathrm{GdMnO}_{3}$} & \multicolumn{3}{|c|}{$\mathrm{CaMnO}_{3}$} \\
\hline & Exp & & & Exp & & \\
\hline & [42] & $V_{0}$ & $V_{\mathrm{rlx}}$ & [43] & $V_{0}$ & $V_{\mathrm{rlx}}$ \\
\hline$a$ & 5.318 & 5.309 & 5.344 & 5.270 & 5.269 & 5.294 \\
\hline$b$ & 5.866 & 5.852 & 5.937 & 5.279 & 5.284 & 5.332 \\
\hline$c$ & 7.431 & 7.425 & 7.426 & 7.456 & 7.457 & 7.496 \\
\hline$x_{R}$ & 0.938 & 0.981 & 0.981 & 0.990 & 0.992 & 0.992 \\
\hline$y_{R}$ & 0.080 & 0.082 & 0.085 & 0.032 & 0.040 & 0.040 \\
\hline$x_{\mathrm{O}_{1}}$ & 0.103 & 0.109 & 0.110 & 0.068 & 0.071 & 0.071 \\
\hline$y_{\mathrm{O}_{1}}$ & 0.471 & 0.465 & 0.465 & 0.493 & 0.488 & 0.487 \\
\hline$x_{\mathrm{O}_{2}}$ & 0.205 & 0.203 & 0.204 & 0.211 & 0.209 & 0.209 \\
\hline$y_{\mathrm{O}_{2}}$ & 0.175 & 0.175 & 0.172 & 0.209 & 0.210 & 0.210 \\
\hline$z_{\mathrm{O}_{2}}$ & 0.550 & 0.552 & 0.552 & 0.530 & 0.538 & 0.536 \\
\hline$d_{z}$ & 1.944 & 1.958 & 1.958 & 1.891 & 1.902 & 1.912 \\
\hline$d_{x 1}$ & 1.910 & 1.920 & 1.923 & 1.896 & 1.911 & 1.923 \\
\hline$d_{x 2}$ & 2.228 & 2.224 & 2.265 & 1.907 & 1.906 & 1.920 \\
\hline$B_{\mathrm{D}}$ & 0.065 & 0.062 & 0.070 & 0.003 & 0.001 & 0.002 \\
\hline$\sigma^{2}$ & 3.883 & 5.915 & 6.776 & 0.281 & 0.377 & 0.213 \\
\hline
\end{tabular}

$1.6 \%$ compared to experimental values (Table I). Consecutively, the octahedron volume was found to be $3 \%$ larger than in [42]. Also the overall volume of the CMO cell and its octahedron volume were found to be overestimated by $1.8 \%$ and $2 \%$, respectively. Such overestimation is known as a characteristic of the GGA functionals in general. The internal parameters for GMO and CMO agree on the contrary very well between the $V_{0}$ and $V_{\mathrm{rlx}}$ calculation schemes (Table I). This motivated again the choice of the experimental lattice constants [44].

Furthermore, both experimental references [42, 43] show the characteristic manganite lattice distortions as described in the introduction. The deviations from the ideal cubic perovskite can be quantified using the two angles, $\alpha$ and $\beta$ (Fig. 2), the Baur's distortion index $\left(B_{\mathrm{D}}\right)$ [45], and the bond angle variance $\left(\sigma^{2}\right)[46] . B_{\mathrm{D}}$ expresses the deviations of the $\mathrm{Mn}-\mathrm{O}$ distances from their mean value. In an undistorted octahedron, the three $\mathrm{Mn}-\mathrm{O}$ distances are equivalent and $B_{\mathrm{D}}$ is zero. The bond angle variance measures the deviation of the twelve $\mathrm{O}-\mathrm{Mn}-\mathrm{O}$ intra-octahedron bond angles $\theta_{i}$ (Fig. 2a) from those $90^{\circ}$ bond angles in an ideal octahedron of the same volume. Thus, $\sigma^{2}$ becomes zero for the ideal octahedron. In contrast to the $\theta_{i}$ the angles $\alpha$ and $\beta$ quantify the mutual 


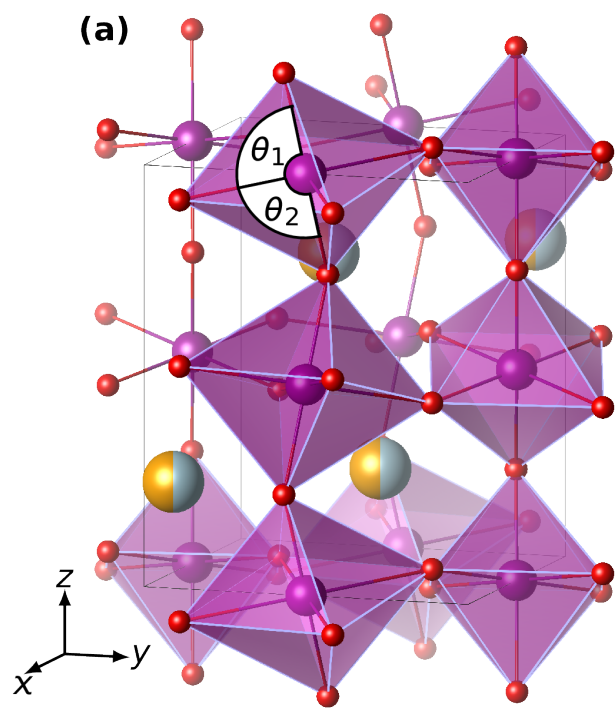

(b)

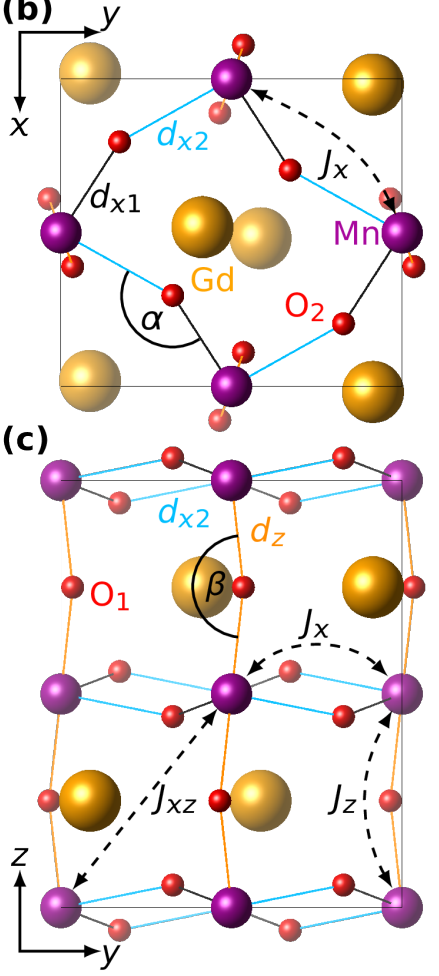

(d)

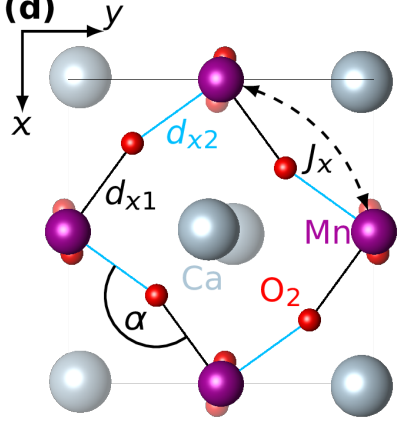

(e)

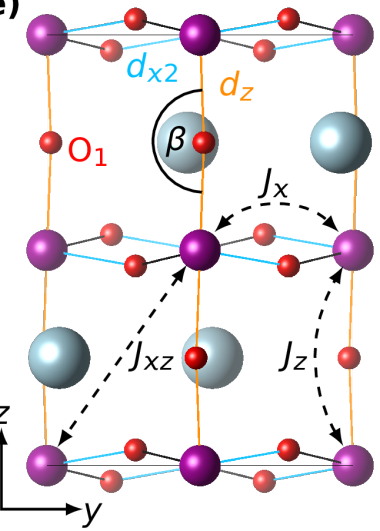

FIG. 2. Structural representation of the Pbnm unit cell of GCMO. (a) Schematic of the 3-dimensional unit cell including the distorted oxygen octahedra. (b),(c) $\mathrm{GdMnO}_{3}$ and (d),(e) $\mathrm{CaMnO}_{3}$. The colored balls depict the $R$ site (mixed colors), Gd (golden), Ca (gray), Mn (violet), and oxygen (red). (b),(d) show the respective top view ( $x y$ plane). (c),(e) feature the side view $(y z$ plane). The structural notation is also indicated for bond length $\mathrm{Mn}-\mathrm{O}$ and the bond angle enclosed in the $\mathrm{Mn}-\mathrm{O}-\mathrm{Mn}$ bond $(\alpha$ and $\beta)$. The three different bond length are noted as $(\mathrm{Mn}-\mathrm{O})_{z}\left(\right.$ orange, $\left.d_{z}\right),(\mathrm{Mn}-\mathrm{O})_{x 1}\left(\mathrm{black}, d_{x 1}\right)$, and $(\mathrm{Mn}-\mathrm{O})_{x 2}$ (blue, $\left.d_{x 2}\right)$. The direction of the magnetic exchange interactions between the Mn sites is pictured as well with dashed arrows. The $\theta_{i}$ in (a) represent two of the intra-octahedron bond angles. Structural figures were prepared with VESTA [12].

tilting of the octahedra.

A similar agreement between experimental and theoretical results, observed for the internal parameters above, is reflected in the distortion index and the bond angle variance as well. We observe an almost similar $B_{\mathrm{D}}$ for GMO but some deviations in $\sigma^{2}$ for GMO and CMO. This shows the advantage of considering both quantities, because they highlight differences in the internal coordinates otherwise not obvious. Nonetheless, we obtain a reduction of the octahedron distortion expected from experimental studies, which is visible in the three $\mathrm{Mn}$ $\mathrm{O}$ bond length - almost similar in $\mathrm{CMO}$, but different in GMO. We conclude that the structural distortions in GMO and CMO [42, 43] are well resembled by the atomic coordinates and the distortion indices determined in our DFT calculations (Table I).

Considering the electronic structure, we obtained an insulating state for both compounds - GMO and CMO [41]. Our calculated Kohn-Sham band gap of GMO without correlation corrections $(0.38 \mathrm{eV})$ agreed with the result by Kováčik et al. [21]. It increases to $1.1 \mathrm{eV}$ with our choice of $U=2 \mathrm{eV}$ [41], although it is still below experimental band gaps obtained from UV absorption spectra of GMO nanoparticles $(2.0 \mathrm{eV})$ [47] or optical measurements $(2.9 \mathrm{eV})[48]$. We observe a strong hybridization between $e_{\mathrm{g}}$ states and $\mathrm{O} p$ states at the valence band maximum of the A-AFM ground state of GMO (see discussion of magnetic ground states below), while the conduction band minimum is formed by a notable mixing between Mn $e_{\mathrm{g}}$ and $t_{2 \mathrm{~g}}$ states [41]. The $e_{\mathrm{g}}$-like valence band width is $0.95 \mathrm{eV}$, in line with the reported $\mathrm{GW}$ band structure [21].

We obtain similar features for the calculated band gap of $\mathrm{CMO}$ obtained with $\mathrm{PBE}+U$, which is $0.92 \mathrm{eV}$. This value is again lower than the experimental band gap $(1.55 \mathrm{eV})$ measured for single crystals of CMO [49].

\section{B. Mapping to a classical Heisenberg model and determination of transition temperatures}

Of all rare-earth elements, Gd stands out due to unique properties. The $\mathrm{Gd}^{3+}$ ions have the largest magnetic moment caused by 7 unpaired spins and show in $\mathrm{GdMnO}_{3}$ the largest observed ordering temperature $(6.5 \mathrm{~K}[50])$ of the $R$ sublattice in all $R \mathrm{MnO}_{3}$ compounds. Neverthe- 
less, the latter ordering temperature following from Gd$\mathrm{Gd}$ magnetic interactions is lower than the one of the $\mathrm{Mn}$ sublattice (45 K [16]), while we can also assume that the transition temperature of the Gd sublattice will not increase with increasing the $\mathrm{Ca}$ concentration. The mean distance between the Gd ions will only increase leading to an even weaker magnetic coupling. Hence, the Gd-Gd interactions are negligible against the magnetic interaction between the Mn ions. We restrict ourselves to the magnetic ordering of the the Mn ions only, while the $f$-states are in the core region. Consequently, when we speak in the following about a magnetic order, we only refer to the orientation of the Mn magnetic moments.

In order to identify the magnetic ground state structures, we took into account ferromagnetic (FM), ferrimagnetic (FiM), and antiferromagnetic structures (Atype, G-type, ... AFM). They are illustrated in Fig. 1 in their minimal required cell, but we needed for the actual calculation a common supercell to accommodate all possible magnetic configurations. We used therefore the 160 atoms supercell described above with fixed lattice constants [16, 44]. The internal coordinates were relaxed only for the FM spin configuration, while they had to be static for other magnetic configurations because of the calculations of the magnetic exchange interactions below. This assumption may slightly bias our results towards a FM ordering (see below) but is a compromise between using the Heisenberg model, much longer computation time, and too many other potential sources of changed materials properties - besides lattice constants, different spin orientations, or later Ca doping.

The total energies are then calculated within this fixed structure for different Mn spin orientations. Those magnetic structures with the lowest total energy resemble the magnetic ground state. The static FM internal coordinates might bias our results slightly towards an FM configuration as the ground state but also the experimental study observed an FM signal over a large Ca concentration range [16], while several other antiferromagnetic ground states could not be finally excluded.

The relative total energies can then be used to verify the experimentally found ground states [5, 43]. We identified the A-AFM and G-AFM as those magnetic structures with the lowest total energy for GMO and CMO, respectively [41]. However, those ground states are not very stable against magnetic variations, since in both cases other magnetic structures are close in energy (FM for GMO, C-AFM for CMO) (see also Fig. 6a).

As mentioned above in the introduction, the $\mathrm{Mn}$ ion appears in two different valence states for GMO $\left(\mathrm{Mn}^{3+}\right)$ and $\mathrm{CMO}\left(\mathrm{Mn}^{4+}\right)$ due to the different valence electron configuration of Gd and Ca. We obtain from our DFT calculations magnetic moments of $3.6 \mu_{\mathrm{B}}$ for $\mathrm{Mn}^{3+}$ (GMO) and $2.7 \mu_{\mathrm{B}}$ for $\mathrm{Mn}^{4+}$ (CMO) [41], which substantially deviate from their integral value of $4 \mu_{\mathrm{B}}$ and $3 \mu_{\mathrm{B}}$, respectively. In GMO, this deviation is caused by the aforementioned hybridization of the Mn states with the oxygen states introducing also a magnetic moment of
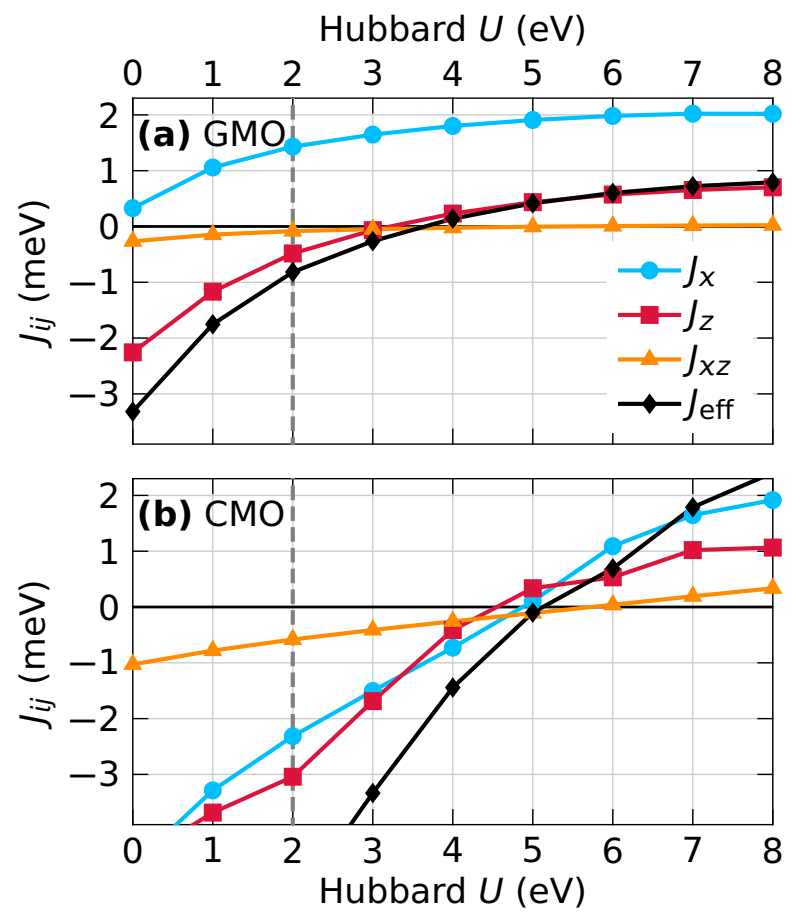

FIG. 3. The three Heisenberg exchange interactions and $J_{\text {eff }}=J_{z}+4 J_{x z}$ in dependence of the correlation treatment in (a) $\mathrm{GdMnO}_{3}$ and (b) $\mathrm{CaMnO}_{3}$. The gray dashed line indicates the choice of $U=2 \mathrm{eV}$ in this work. See Fig. 2 for the visualization of the three magnetic coupling directions.

$0.06 \mu_{\mathrm{B}}$ at the oxygen ions [41]. Our observed local magnetic moment of $\mathrm{CMO}$ is in line with its experimental value of $2.665 \mu_{\mathrm{B}}$ [9], while we did not find any experimental value of the local magnetic moment of $\mathrm{Mn}^{3+}$ in GMO. Nevertheless, the $3.6 \mu_{\mathrm{B}}$ for $\mathrm{Mn}^{3+}$ in GMO agree with earlier numerical calculations including hybrid functionals [21].

In addition to the magnetic ground state, we will need below for a full description of the magnetic phase diagram of GCMO also the corresponding finite temperature characteristics - namely the critical transition temperatures. The latter can be derived on basis of the classical Heisenberg model from DFT total energies. Therefore, the total energies are mapped onto a Hamiltonian of the form

$$
H=-\frac{1}{2} \sum_{i \neq j} J_{i j} \boldsymbol{S}_{i} \cdot \boldsymbol{S}_{j}
$$

The parameters $J_{i j}$ are $J_{x}$ (in-plane interaction), $J_{z}$ (outof-plane interaction), and $J_{x z}$ (interaction along the cell diagonal), if ( $i j)$ describes a corresponding pair of atoms as indicated in Fig. 2. We can also define an effective out-of-plane interaction $J_{\text {eff }}=J_{z}+4 J_{x z}$, which can characterize the tendency to an out-of-plane antiferromagnetic order [41]. The sums in (1) run over all sites $i$ with the interaction sites corresponding to each $J_{i j}$. Positive (negative) $J_{i j}$ correspond to FM (AFM) coupling. The spin moment $S$ in (1) equals to 2 for $\mathrm{Mn}^{3+}$ (4 unpaired 
electrons/2) and 3/2 for $\mathrm{Mn}^{4+}$ (3 unpaired electrons/2). This kind of Hamiltonian was used to study magnetic properties of GMO before [21] and has an advantage over many other studies on magnetic properties of $R \mathrm{MnO}_{3}$ being restricted only to the nearest $\mathrm{Mn}$ neighbors exchange couplings.

The three magnetic exchange parameters can be then obtained by mapping total energies of different spin orientations (Fig. 1) onto the Heisenberg Hamiltonian in (1). This results in an over-determined set of equations, which is solved with a linear least square fit [41]. The ferrimagnetic configuration FiM (Fig. 1) was used as the reference energy $E_{0}$ inspired by [21].

At this point, we want to emphasize again the importance of a correct electronic correlation treatment in our materials. Our determined magnetic exchange interactions vary strongly with increasing $U$ parameters (Fig. 3). We even obtained with the PBE exchange correlation functional, without $U$ correction, for GMO a wrong Gtype AFM ground state. The out-of-plane contribution characterized by $J_{\text {eff }}$ dominates the in-plane interaction (Fig. 3a). Only when $U$ is increased to be around $2 \mathrm{eV}$, the in-plane exchange becomes stronger and leads with $J_{x}>0$ and $J_{\text {eff }}<0$ to the correct known A-AFM phase [41]. Increasing $U$ further results in an FM order: the magnitude of $J_{\text {eff }}$ decreases and it turns positive (ferromagnetic) for $U \gtrsim 4 \mathrm{eV}$ (Fig. 3a). This observation matches well with the potential instability against a FM state found for GMO based on the total energy calculations. The energy difference between the FM and A-AFM states is $\sim 4 \mathrm{meV}$ (Fig. 6).

For CMO, the situation is slightly different. The three exchange interactions are negative for plain PBE (see Fig. 3b) and only become positive for $U>5 \mathrm{eV}$, which is far above a reasonable value considering other materials properties. The strong competition between the three exchange parameters for $U<4 \mathrm{eV}$ leads to the G-type AFM phase.

Finally, we want to assess the magnetic transition temperature (either $T_{\mathrm{N}}$ for AFM phases or $T_{\mathrm{C}}$ for FM and FiM) and used our own Monte-Carlo (MC) code [51] together with the Heisenberg Hamiltonian (1). Therein, we use a large cluster of $16 \times 16 \times 16$ times the primitive unit cell (a total volume of about $100 \AA^{3}$ ). Periodic boundary conditions are also considered. The thermal equilibrium was firstly assumed to be reached after $60000 \mathrm{MC}$ steps. Another 20000 steps are then used in the thermal averaging. We started from a high temperature of $500 \mathrm{~K}$ and cooled down the GCMO samples in steps of $3 \mathrm{~K}$. The transition temperatures are later extracted from the temperature dependence of three quantities - the magnetic susceptibility, saturation magnetization, and the heat capacity. The calculated exchange interactions are used for the initial system configuration. An ordering temperature of $42 \mathrm{~K}$ was obtained for GMO, which matches perfectly the experimental value of $40 \mathrm{~K}[5,50]$. In contrast, a hybrid functional calculation led to a little overestimation of $T_{\mathrm{N}}$ by about $20 \mathrm{~K}$ [21]. For CMO, a $T_{\mathrm{N}}$ of $96 \mathrm{~K}$ was obtained, which is in the same range as the experimentally observed $T_{\mathrm{N}}$ of $125 \mathrm{~K}$ [52].

We conclude that our computational setup and the procedures in order to obtain the magnetic ground state and the magnetic transition temperatures produce results in good agreement with available experimental data. Therefore, we have a proper basis for the study of the complete series of intermixed rare-earth and alkaline earth manganites.

\section{PHASE DIAGRAM FOR GCMO}

Using the orthorhombic structure for all $\mathrm{Ca}$ concentrations $x$ in the $\mathrm{Gd}_{1-x} \mathrm{Ca}_{x} \mathrm{MnO}_{3}$ series, we want to take into account the disordered character of this solid solution. Besides the above mentioned KKR-CPA method, another possible way is to average the $(\mathrm{Gd}, \mathrm{Ca})$ sublattice occupancy over different structures within a large supercell with $N$ functional units. Such method is impractical because one has in general to average about too many configurations, even if symmetry arguments are used to find potentially different configurations. In order to circumvent the problem, we used the special quasi-random structure (SQS) method [20] for the (Gd,Ca) sublattice. SQS takes into account the random nature of alloys by choosing the occupation of the internal coordinates inside a supercell in such a way that the pair and multisite correlations mimic as much as possible those of a random substitutional alloy. The multi-site correlations in the SQS candidates are then taken into account and compared to the random distribution up to a defined cutoff radius. The resulting structure based on these constraints is not necessarily a fully disordered structure, but a good approximation to the real solid solution character of the material, as follows from the correlation functions of the SQS [41].

To the best of our knowledge, it is the first time that the SQS method is applied to such manganites. The generation of the SQS cells was carried out using a MonteCarlo annealing loop, as implemented in the MCSQS routine of the ATAT package [53]. We forced the axis orthogonality in the SQS cells, which kept the distance between the Mn sites and mostly the angles between them constrained throughout the GCMO series. In this way, we can keep the same definition of the three aforementioned exchange coupling constants defined in section IIB for the following comparison of magnetic properties throughout the concentration range.

Nevertheless, the concentration $x$ cannot be chosen continuously between $0 \%$ to $100 \%$ but depends on the size of the 160 atoms supercell. Therefore, the smallest concentration step used in the simulation can be only $1 / 2^{3}=1 / 8$ and we performed all calculations for the concentrations $x=0,1 / 8,1 / 4, \ldots, 7 / 8,1$.

Many SQS reported in the literature are obtained by matching only pair correlations. In this work, we include also higher order correlations of the random structure. 
Pair clusters are taken up to the $5^{\text {th }}$ nearest neighbor, triplet and quadruplet clusters are included up to the $4^{\text {th }}$ nearest neighbor. Only for $x=0.5$, the SQS structure fully resembles a completely disordered system with zero correlation functions (see [41]). The other correlation function results and structural details of the SQS are collected in [41].

Although we include also disordered Ca doping in our study, there are still some limitations. A careful consideration of the coupling between spin, charge, lattice and orbital degress of freedom should be done, but this is far from trivial, especially with our large supercells. On top of the additional plethora of relaxations for the AFM structures, different charge or orbital ordering states should be taken into account - maybe even in dependence of different $\mathrm{Ca} / \mathrm{Gd}$ distributions. Such a number of correlations is beyond the scope of this work and we restrict therefore ourselves to the coupling between the variation of lattice constants, the disorder-like Ca concentration within an SQS cell, and the magnetic states considered for static internal parameters.

The experimental lattice parameter $a$ and $c / \sqrt{2}$ vary only little with a slight maximum for $x=0.5$. Only $b$ decreases strongly until $x=0.5$ and follows afterwards $a$ and $c / \sqrt{2}[16,44]$. That means that the unit cell volume of GCMO contracts with increasing Ca concentration $x$, which results in a gain of $\mathrm{Mn}^{4+}$ content. Such volume contraction in manganites is commonly explained based on ionic radii, because the ionic radius of 6 -fold coordinated $\mathrm{Mn}^{4+}(0.53 \AA)$ is smaller compared to that of $\mathrm{Mn}^{3+}$ $(0.645 \AA)$ [54]. But at the same time, the ionic radius of the introduced $\mathrm{Ca}^{2+}$ ions (12-fold coordinated: $1.34 \AA$ ) is larger than that of the substituted $\mathrm{Gd}^{3+}$ ions' (9-fold coordinated: $1.107 \AA$ ). The 9-fold coordination is a good estimate due to the strong distortions in GMO (see Fig. 2 ). For $\mathrm{Ca}^{2+}$, the coordination is as well not clearly $12-$ fold but also the ionic radius of 10 -fold coordinated $\mathrm{Ca}^{2+}$ $(1.23 \AA)$ is still larger than the one of $\mathrm{Gd}^{3+}$ and the above statement holds true. Despite all these aspects, an overall volume contraction is still observed together with less distortions in the Mn octahedra. Thus, a simple analysis based on ionic radii alone is not possible but we see that several structural aspects are intertwined: ionic radii, site coordinate, doping concentration, and atomic bonding.

We tracked the distortion of the Mn octahedra via the variation of bond angles and bond lengths in all GCMO compounds. Therefore, we calculated the mean value of all present bond lengths (angles) inside the relaxed SQS cells (Fig. 4). The changes of the bond lengths match the behavior of the experimental lattice constants in having distinct changes at $x=0.5$ (Fig. 4a), which holds also true for the Mn-O-Mn bond angles (Fig. 4b). The resulting distortion indices, $B_{\mathrm{D}}$ and $\sigma^{2}$ (not shown), decrease linearly with increasing $x$. Only at $x=5 / 8$, both indices show an anomaly, which follows exactly the peculiar deviation of the cell parameters at the aforementioned concentration.

The calculated density of states of the GCMO series
Ca Concentration (\%)
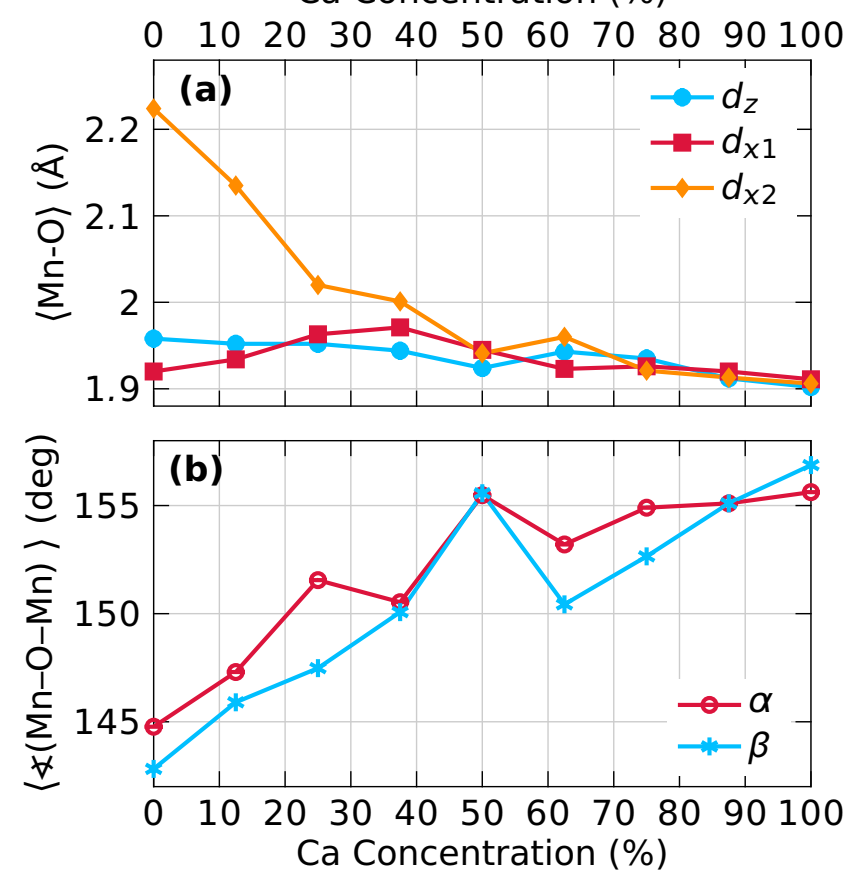

FIG. 4. The variation of (a) the three $\mathrm{Mn}-\mathrm{O}$ bond lengths and (b) the $\mathrm{Mn}-\mathrm{O}-\mathrm{Mn}$ bond angles averaged over the SQS bond lengths and angles, respectively, with respect to the $\mathrm{Ca}$ concentration in the whole GCMO series. See Fig. 2 for the visualization of both structural properties.

shows a half-metallic-like behavior, that means the DOS being metallic in the majority spin channel, but having a band gap of $1 \mathrm{eV}$ to $1.5 \mathrm{eV}$ in the minority spin channel (see [41]). A similar result was shown for $\mathrm{La}_{1-x} \mathrm{Ca}_{x} \mathrm{MnO}_{3}$ [55], where the insulating character of the density of states was only recovered by localizing the additional electron (hole) in the system.

The magnetic ground state structures for GCMO are determined, as in section II B, for the SQS at every concentration as well. The number of relevant magnetic exchange interactions remains also the same, $J_{x}, J_{z}$, and $J_{x z}$ (Fig. 2), due to the conserved Mn distances in the supercells. We only vary $S$ as the mean value of the spin moments, which corresponds to the respective $\mathrm{Ca}$ concentration

$$
S_{x}=(1-x) S_{\mathrm{Mn}^{3+}}+x S_{\mathrm{Mn}^{4+}},
$$

with $S_{\mathrm{Mn}^{3+}}=2$ and $S_{\mathrm{Mn}^{4+}}=3 / 2$. In the case of partial occupation of $\mathrm{Gd}$ sublattice $(0<x<1)$, the distinction between $\mathrm{Mn}^{3+}$ and $\mathrm{Mn}^{4+}$ is ignored in all our calculations. They are treated at the same footing as effective $\mathrm{Mn}$ ions with concentration dependent valence states taking a value of $3+$ at $x=0$ and $4+$ in $x=1$. Following the experimental literature $[9,10,16]$, we can distinguish three different doping regimes: hole doping for $x<1 / 2$, middle doping region for $1 / 2 \leq x<7 / 8$, and electron doping $x \geq 7 / 8$. For $x<0.5$ the lattice parameter $b$ is considerably larger than $a$, but they become 


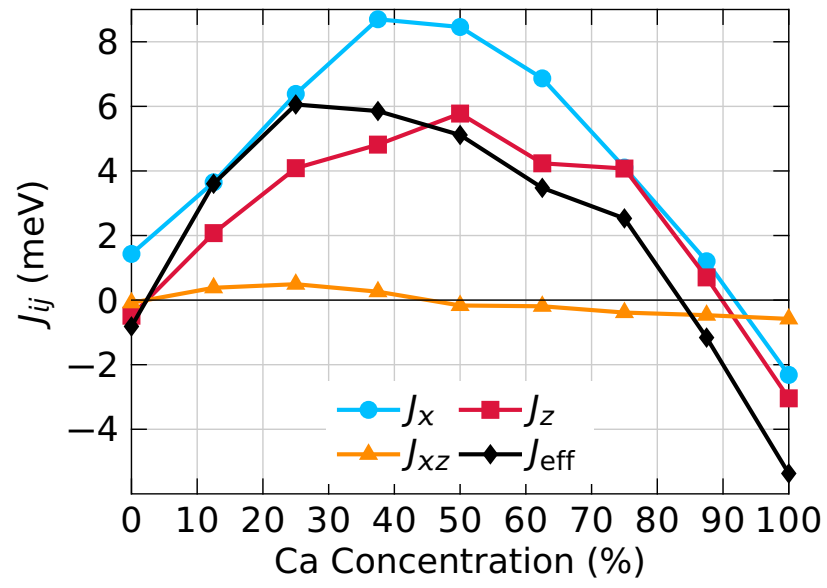

FIG. 5. The calculated Heisenberg exchange interactions in $\mathrm{Gd}_{1-x} \mathrm{Ca}_{x} \mathrm{MnO}_{3}$ following equations (1) and (2), and $J_{\text {eff }}=$ $J_{z}+4 J_{x z}$. See Fig. 2 for the visualization of the three magnetic coupling directions. $U=2 \mathrm{eV}$ is used in the underlying electronic structure calculations [41].

equal for $x \geq 0.5[16]$. Connected to the change in the lattice constants, all $\mathrm{Mn}-\mathrm{O}$ bond lengths become nearly equal beginning from $x=0.5$, while the tilting angle $\alpha$ becomes practically independent on the concentration in this region (Fig. 4).

\section{A. Hole doping: $x<1 / 2$}

Adding $\mathrm{Ca}$ to $\mathrm{GdMnO}_{3}$ introduces a hole in the vicinity of the $\mathrm{Ca}^{2+}$ ion, which is compensated by an additional electron from $\mathrm{Mn}$ - the already mentioned $\mathrm{Mn}^{4+}$ is created. This process causes a transition of the AAFM phase to a FM state in the concentration range $0<x<0.5$, experimentally verified by Beiranvand et al. [16]. Their temperature dependent SQUID measurements show in addition a negative magnetization at $x=0.1$ and $T<20 \mathrm{~K}$, which they mainly attributed to the Gd spins - orienting themselves antiparallel to the direction of the Mn spins. This ferrimagnetic coupling was firstly proposed for $x=0.3$ [56] and thereafter generalized for $x<1 / 2[16,57]$ of GCMO. The same FM phase transition is obtained in our calculation with the SQS structure at $x=1 / 8$. Before at $x=0$, the FM state has not the lowest total energy but its energy difference to the A-AFM state is rather small (see Fig. 6a). The increase of the Ca concentration to $x=1 / 8$ turns the sign of the total energy difference and enhances it strongly: the FM state is $(29 \mathrm{meV})$ below the A-AFM state and even more for $x=1 / 4$ (see Fig. 6a). This first transition is connected with a strong increase of the inplane exchange parameter $\left(J_{x}\right)$ to $3.6 \mathrm{meV}$ and an AFM to FM change of the out-of-plane exchange interactions - visible in $J_{\text {eff }}$ (Fig. 5). The latter goes from negative to positive. An A-AFM state is only realized for $J_{x}>0$ and $J_{\text {eff }}<0$. This variation in the magnetic coupling
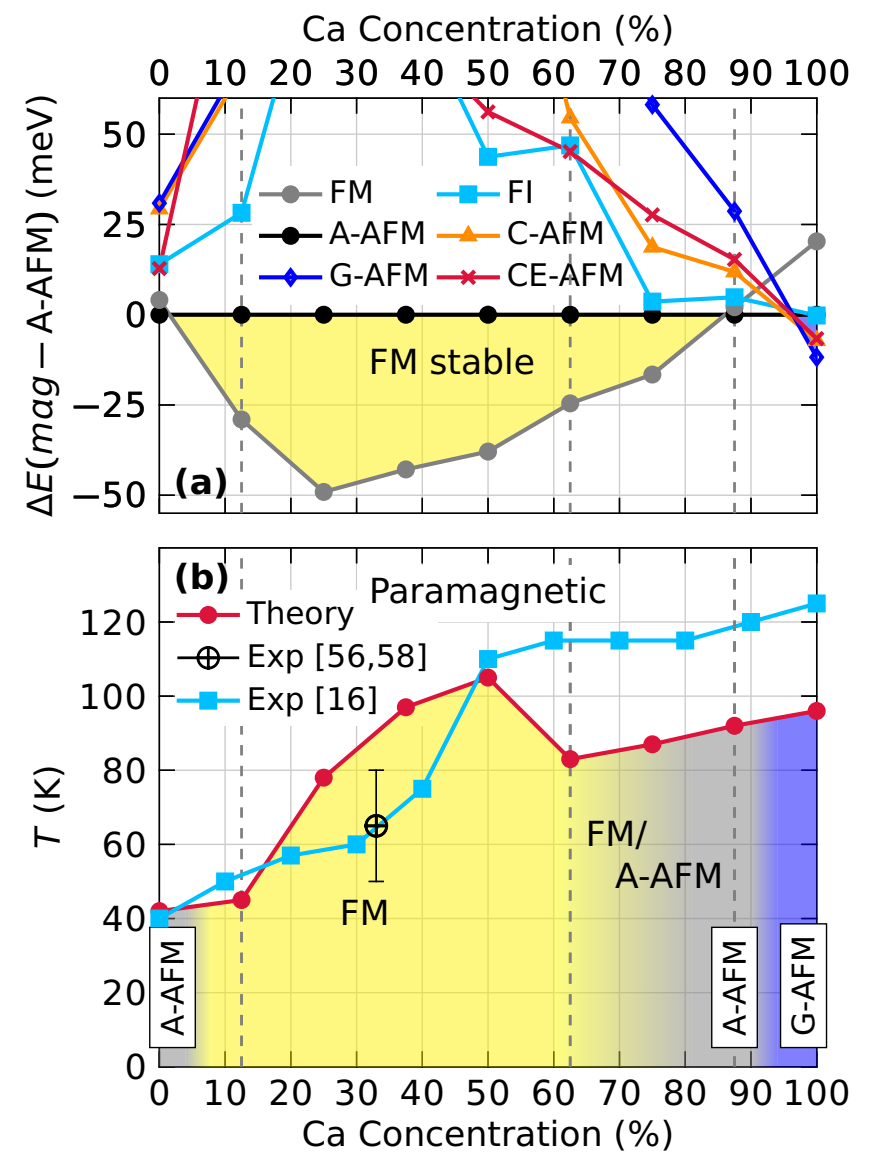

FIG. 6. (a) The concentration dependent total energy landscape of the most relevant magnetic ground state structures $(m a g)$ depicted in Fig. 1. The energy differences $\Delta E(m a g-$ A-AFM) are calculated with respect to the A-AFM state. That magnetic state $(\mathrm{mag})$, which has the lowest $\Delta E$, is the most stable one. (b) The theoretical magnetic phase diagram of $\mathrm{Gd}_{1-x} \mathrm{Ca}_{x} \mathrm{MnO}_{3}$. The critical temperatures (red circles) were determined via the Monte Carlo simulations, while the magnetic phases were identified from the minimal total energy. The measured critical temperatures from Ref. [16] (blue squares) and for $x=1 / 3$ from Ref. [56, 58] (black $\oplus$ with error bars) are shown for comparison. Above the critical temperature, we expect a paramagnetic state. The region marked with FM/A-AFM identifies the concentration range, where the total energy difference of the FM and A-AFM magnetic phase is below $25 \mathrm{meV}$. Dashed lines mark qualitative changes of the magnetic ordering.

strength does not only result in the A-AFM to FM transition but also in an increased Curie temperature until $x=1 / 2$ (see Fig. 6b), which qualitatively matches the experimental measurements of a FM order in the whole hole-doped region of GMO $[16,56,58]$. Such magnetic alteration could be attributed to the progressive increase of the $\mathrm{Mn}-\mathrm{O}-\mathrm{Mn}$ bond angle with the doping level, as well as the drastic shrink of the in-plane $(\mathrm{Mn}-\mathrm{O})_{x 2}$ bond length (Fig. 4). The Mn-O-Mn bond angle was, e.g., reported for $x=1 / 4$ as $149.7^{\circ}$ [59], which is the average of our two calculated angles, $147^{\circ}$ and $151.7^{\circ}$. Ac- 
companied with the decrease of the cell parameter $b$, the overall cell distortion diminishes and we can conclude that the $\mathrm{Ca}$ induced magnetic transformation is mainly triggered by the reduction of the Jahn-Teller distortion. The disagreement between the measured and calculated transition temperatures in Fig. 6b could have, besides the known problems of $T_{\mathrm{C}}$ calculations, several different explanations: Lattice imperfections like vacancies, in particular at the oxygen sublattice, might cause significant changes in the magnetic properties as observed for other oxides, like $\mathrm{SrCoO}_{3}$ [60] or $\mathrm{Sr}_{2} \mathrm{FeMoO}_{6}$ [61]. In addition, differences between experiments and theoretical simulations may be connected to the fact, that an ideal periodic crystal is assumed in the simulation, while the samples are polycrystalline. Furthermore, a more complicated magnetic structure might occur for $x=0.33$ (canted antiferromagnetic) instead of a simple ferromagnetic state as supposed by Snyder et al. [56].

\section{B. Half occupied: $1 / 2 \leq x<7 / 8$}

In the mid-doped region for $x \sim 0.5$, our Monte-Carlo simulation determined a transition temperature of $105 \mathrm{~K}$ - close to the reported bulk temperature (107 K). The exchange coupling $J_{x z}$ becomes negative already for $x=0.5$ (see Fig. 5), but the FM order still remains the ground state. With increasing Ca concentration, the energy difference between the FM and A-AFM order becomes gradually smaller (see Fig. 6).

The Mn-O-Mn bond angles become equivalent - both are $155^{\circ}$ (Fig. 4b) and all $\mathrm{Mn}-\mathrm{O}$ distances decrease to roughly the similar distance (Fig. $4 \mathrm{a}$ ). Hence, the octahedron distortion becomes less pronounced than before, which hints also to the ferromagnetic order due to the double exchange mechanism following from the different valency of the Mn ions.

The concentration $x=0.5$ marks the transition to an antiferromagnetic ground state in the experimental phase diagram [16]. Due to missing neutron diffraction data the particular type of antiferromagnetic order is not known from experiments. The calculation still leads to a ferromagnetic ground state for this concentration, but charge order $(\mathrm{CO})$ is observed experimentally. Thus, in a next step of our investigations the concentration range $1 / 2 \leq x \leq 7 / 8$ has to be investigated with inclusion of charge order phenomena. This room temperature CO state makes the mid-doped concentration range not only most interesting for technical applications but might have also an important role in the stabilization of the AFM order, which was discussed, e.g., for $\mathrm{La}_{0.5} \mathrm{Ca}_{0.5} \mathrm{MnO}_{3}$ [62]. The latter compound was reported to be a ferromagnetic metal due to double exchange coupling but becomes a antiferromagnetic insulator for temperatures $T \lesssim 195 \mathrm{~K}$. The authors of Ref. [62] suggest that the latter AFM phase transition coincides with a charge ordering transition, which suppresses the ferromagnetism and stabilizes the AFM order.
Another potential stabilization mechanism of the AFM order was proposed for $\mathrm{Pr}_{1-x} \mathrm{Ca}_{x} \mathrm{MnO}_{3}$ (PCMO). Its magnetic order at $x=0.5$ is rather maintained by the presence of the so called Zener polarons, because a stabilization of a CE-type AFM order by means of the $\mathrm{CO}$ could be excluded based on single-crystal neutron diffraction measurements $[11,63]$. This phenomenon results from trapped electrons between the two Mn sites causing a valence of $3.5+$ in the neighboring $\mathrm{Mn}$ ions instead of the natural valence of $3+$ or $4+$, respectively.

An analogous argument was given by García et al. [64] using a ferromagnetic Kondo lattice model. Therewith, they demonstrate that the formation of magnetic polarons is an important ingredient in the description of systems with correlated spin-charge degrees of freedom. This correlation is induced from the strong competition between double exchange and superexchange mechanisms. The signature of such coexisting spin and charge ordering could be as well obtained from DFT calculations. However, such phenomena require much more computational effort, i.e. different charge patterns have to be checked at each $x$ concentration and for all considered magnetic orders in this study.

Adding then more $\mathrm{Ca}$ does not change the qualitative picture. The FM order remains still the lowest magnetic ground state structure and the corresponding Curie temperatures are still high $(>80 \mathrm{~K})$ (Fig. 6). However, the total energy difference to the A-AFM order is strongly reduced and at $x=3 / 4$, the ferrimagnetic (FiM) order (Fig. 1) starts to compete for the lowest total energy. Here, $J_{x}$ and $J_{z}$ are equivalent, while the AFM coupling $J_{x z}$ increases (Fig. 5).

\section{Electron doping: $7 / 8 \leq x<1$}

The last doping regime represents essentially $\mathrm{CaMnO}_{3}$ doped with few percent $\mathrm{Gd}$ ions, which adds excess electrons from $\mathrm{Gd}^{3+}$. Therein, the A-AFM overcomes the ferromagnetic order (Fig. 6) because the strength of the magnetic coupling decreases and the effective out-ofplane interaction becomes negative again (Fig. 5). All three exchange parameters are of a similar magnitude, $J_{x}=1.20 \mathrm{meV}, J_{z}=0.70 \mathrm{meV}$, and $J_{x z}=-0.47 \mathrm{meV}$. This energetic competition reduces also the total energy of other magnetic structures and makes them more likely. The smallest energy difference is realized by the FiM state (Fig. 6a) but also G-AFM and C-AFM show very small energy differences and might become more relevant. In particular, the C-type AFM order is also assumed for $x=0.8$ by Beiranvand et al. [16] but remains in our calculation at $x=7 / 8$ still $11 \mathrm{meV}$ higher in energy than the A-AFM.

This variation of potential antiferromagnetic structures offers a large playground for the study of basic principles in magnetic coupling and the resulting ground states. Hence, the electron doping concentration range $7 / 8 \leq x<1$ is, in particular, scientifically interesting, 
because the experimental results vary a lot: Beiranvand et al. [16] did not detect an CO state for $x>0.7$, but Khan et al. [65] found that it should coexist with OO simultaneously at $x=0.85$ and be even very robust against external influence, since the application of a magnetic field up to $15 \mathrm{~T}$ between $5 \mathrm{~K}$ to $300 \mathrm{~K}$ did not annihilate the charge ordering. In addition, colossal magneto resistance was detected at $0.8<x<0.9$ and $T=10 \mathrm{~K}$, in the boundary between the CO-AFM insulating state and the cluster-glass (CG) state [16]. The latter was explained by the simultaneous existence of FM metallicity and an AFM insulating state [66].

\section{SUMMARY}

We investigated theoretically the magnetic phase diagram of the whole GCMO series for the first time and observed a good qualitative agreement with the available experimental data [16]. We identified the different magnetic ground states being mainly a ferromagnetic coupling between the Mn magnetic moments with instabilities towards ferrimagnetic or A-type antiferromagnetic spin orientations. The calculated magnetic transition temperatures agree well with the experimentally derived ones but show a systematic difference to experiment for $x>60 \%$. This might be connected with the unstable an- tiferromagnetic coupling between the Mn ions observed in the same concentration range. In summary, we obtained a rather good agreement between the numerical calculations based on the special quasi random structures simulating the miscibility of the GCMO series and the earlier experimental study of the whole concentration range [16]. Several concepts remain still unknown for GCMO and need to be carefully examined e.g.: Does GCMO favor a collinear or non-collinear magnetism? Which combination of spin, charge and orbital ordering is likely to occur in GCMO? What is the effect of strain or defects on the magnetic phase diagram. Thereby, our study lays a basis for further experimental and theoretical studies of the solid solution rare earth manganites and in particular GCMO.

\section{ACKNOWLEDGMENTS}

This publication was funded by the German Research Foundation within the Collaborative Research Centre 762 (Projects No. A4 and No. B1) and the German Academic Research Council (Project number 57348127). The Jenny and Antti Wihuri Foundation is acknowledged for financial support. The computer resources of the Finnish IT Centre for Science (CSC), project No. 2000643, are acknowledged.
[1] J. Mannhart and D. G. Schlom, Science 327, 1607 (2010).

[2] S. Ismail-Beigi, F. J. Walker, A. S. Disa, K. M. Rabe, and C. H. Ahn, Nat. Rev. Mater. 2, 17060 (2017).

[3] R. von Helmolt, J. Wecker, B. Holzapfel, L. Schultz, and K. Samwer, Phys. Rev. Lett. 71, 2331 (1993).

[4] M. B. Salamon and M. Jaime, Rev. Mod. Phys. 73, 583 (2001).

[5] T. Kimura, S. Ishihara, H. Shintani, T. Arima, K. T. Takahashi, K. Ishizaka, and Y. Tokura, Phys. Rev. B 68, 060403 (2003).

[6] C. Zener, Phys. Rev. 82, 403 (1951).

[7] P. W. Anderson and H. Hasegawa, Phys. Rev. 100, 675 (1955).

[8] M. Fiebig, J. Phys. D: Appl. Phys. 38, R123 (2005).

[9] E. O. Wollan and W. C. Koehler, Phys. Rev. 100, 545 (1955).

[10] J. B. Goodenough, Phys. Rev. 100, 564 (1955).

[11] M. C. Langner, S. Zhou, G. Coslovich, Y.-D. Chuang, Y. Zhu, J. S. Robinson, W. F. Schlotter, J. J. Turner, M. P. Minitti, R. G. Moore, W. S. Lee, D. H. Lu, D. Doering, P. Denes, Y. Tomioka, Y. Tokura, R. A. Kaindl, and R. W. Schoenlein, Phys. Rev. B 92, 155148 (2015).

[12] K. Momma and F. Izumi, J. Appl. Crystallogr. 44, 1272 (2011).

[13] T. Kimura, G. Lawes, T. Goto, Y. Tokura, and A. P. Ramirez, Phys. Rev. B 71, 224425 (2005).

[14] Y. Yamasaki, H. Sagayama, N. Abe, T. Arima, K. Sasai, M. Matsuura, K. Hirota, D. Okuyama, Y. Noda, and Y. Tokura, Phys. Rev. Lett. 101, 097204 (2008).
[15] A. Pimenov, A. A. Mukhin, V. Y. Ivanov, V. D. Travkin, A. M. Balbashov, and A. Loidl, Nat. Phys. 2, 97 (2006).

[16] A. Beiranvand, J. Tikkanen, H. Huhtinen, and P. Paturi, J. Alloys Comp. 720, 126 (2017).

[17] J. M. Cadogan, D. H. Ryan, M. Napoletano, P. Riani, and L. M. D. Cranswick, J. Phys.: Condens. Matter 21, 124201 (2009).

[18] M. Hoffmann, A. Marmodoro, E. Nurmi, K. Kokko, L. Vitos, A. Ernst, and W. Hergert, Phys. Rev. B 86, 094106 (2012).

[19] The KKR-CPA method is implemented in our code HUTSEPOT [67]. Calculations for GCMO with this code are on the way, but not completed up to now.

[20] A. Zunger, S.-H. Wei, L. G. Ferreira, and J. E. Bernard, Phys. Rev. Lett. 65, 353 (1990).

[21] R. Kováčik, S. S. Murthy, C. E. Quiroga, C. Ederer, and C. Franchini, Phys. Rev. B 93, 075139 (2016).

[22] W. S. Ferreira, E. Moreira, and N. F. Frazão, Braz. J. Phys. 48, 126 (2018).

[23] C. Xu, Y. Li, B. Xu, J. Íñiguez, W. Duan, and L. Bellaiche, Adv. Funct. Mater. 27, 1604513 (2017).

[24] D. Mekam, S. Kacimi, M. Djermouni, M. Azzouz, and A. Zaoui, Results Phys. 2, 156 (2012).

[25] H. R. Aliabad, Z. Barzanuni, S. R. Sani, I. Ahmad, S. Jalali-Asadabadi, H. Vaezi, and M. Dastras, J. Alloys Comp. 690, 942 (2017).

[26] N. S. Fedorova, Y. W. Windsor, C. Findler, M. Ramakrishnan, A. Bortis, L. Rettig, K. Shimamoto, E. M. Bothschafter, M. Porer, V. Esposito, Y. Hu, A. Alberca, T. Lippert, C. W. Schneider, U. Staub, 
and N. A. Spaldin, ArXiv e-prints (2018), provided by the SAO/NASA Astrophysics Data System, arXiv:1805.02172.

[27] S. Bhattacharjee, E. Bousquet, and P. Ghosez, Phys. Rev. Lett. 102, 117602 (2009).

[28] U. Aschauer and N. A. Spaldin, Appl. Phys. Lett. 109, 031901 (2016).

[29] U. Aschauer, R. Pfenninger, S. M. Selbach, T. Grande, and N. A. Spaldin, Phys. Rev. B 88, 054111 (2013).

[30] S. Keshavarz, Y. O. Kvashnin, D. C. M. Rodrigues, M. Pereiro, I. Di Marco, C. Autieri, L. Nordström, I. V. Solovyev, B. Sanyal, and O. Eriksson, Phys. Rev. B 95, 115120 (2017).

[31] N. Bondarenko, Y. Kvashnin, J. Chico, A. Bergman, O. Eriksson, and N. V. Skorodumova, Phys. Rev. B 95, 220401 (2017).

[32] J. Klarbring and S. I. Simak, Phys. Rev. B 97, 024108 (2018).

[33] P. E. Blöchl, Phys. Rev. B 50, 17953 (1994).

[34] G. Kresse and D. Joubert, Phys. Rev. B 59, 1758 (1999).

[35] G. Kresse and J. Furthmüller, Comp. Mater. Sci. 6, 15 (1996).

[36] G. Kresse and J. Furthmüller, Phys. Rev. B 54, 11169 (1996).

[37] J. P. Perdew, K. Burke, and M. Ernzerhof, Phys. Rev. Lett. 77, 3865 (1996).

[38] J. P. Perdew and Y. Wang, Phys. Rev. B 45, 13244 (1992).

[39] J. P. Perdew and A. Zunger, Phys. Rev. B 23, 5048 (1981).

[40] S. L. Dudarev, G. A. Botton, S. Y. Savrasov, C. J. Humphreys, and A. P. Sutton, Phys. Rev. B 57, 1505 (1998).

[41] See Supplemental Material at [URL will be inserted by publisher] for details about the computational setup, validation of the choice of exchange-correlation functionals, magnetic moments, density of states, magnetic exchange parameters, and the special quasi random structures.

[42] T. Mori, N. Kamegashira, K. Aoki, T. Shishido, and T. Fukuda, Mater. Lett. 54, 238 (2002).

[43] M. E. Melo Jorge, M. R. Nunes, R. Silva Maria, and D. Sousa, Chem. Mater. 17, 2069 (2005).

[44] Experimental lattice constants were provided by Wihuri Physical Laboratory, University of Turku, Finland.

[45] W. H. Baur, Acta Crystallogr. B 30, 1195 (1974).

[46] K. Robinson, G. V. Gibbs, and P. H. Ribbe, Science 172, 567 (1971).

[47] X. L. Wang, D. Li, T. Y. Cui, P. Kharel, W. Liu, and Z. D. Zhang, J. Appl. Phys. 107, 09B510 (2010).

[48] P. Negi, G. Dixit, H. M. Agrawal, and R. C. Srivastava, J. Supercond. Nov. Magn. 26, 1611 (2013).

[49] N. N. Loshkareva, L. V. Nomerovannaya, E. V. Mostovshchikova, A. A. Makhnev, Y. P. Sukhorukov, N. I. Solin, T. I. Arbuzova, S. V. Naumov, N. V. Kostromitina, A. M. Balbashov, and L. N. Rybina, Phys. Rev. B 70, 224406 (2004).

[50] J. Hemberger, S. Lobina, H.-A. Krug von Nidda, N. Tristan, V. Y. Ivanov, A. A. Mukhin, A. M. Balbashov, and A. Loidl, Phys. Rev. B 70, 024414 (2004).

[51] G. Fischer, M. Däne, A. Ernst, P. Bruno, M. Lüders, Z. Szotek, W. Temmerman, and W. Hergert, Phys. Rev. B 80, 014408 (2009).

[52] Z. Zeng, M. Greenblatt, and M. Croft, Phys. Rev. B 59, 8784 (1999).
[53] A. van de Walle, P. Tiwary, M. de Jong, D. Olmsted, M. Asta, A. Dick, D. Shin, Y. Wang, L.-Q. Chen, and Z.-K. Liu, Calphad 42, 13 (2013).

[54] All ionic radii were taken from http://abulafia.mt.ic. ac.uk/shannon/, which is based on Ref. [68].

[55] R. Korotana, G. Mallia, Z. Gercsi, L. Liborio, and N. M. Harrison, Phys. Rev. B 89, 205110 (2014).

[56] G. J. Snyder, C. H. Booth, F. Bridges, R. Hiskes, S. DiCarolis, M. R. Beasley, and T. H. Geballe, Phys. Rev. B 55, 6453 (1997).

[57] O. Pena, M. Bahout, K. Ghanimi, P. Duran, D. Gutierrez, and C. Moure, J. Mater. Chem. 12, 2480 (2002).

[58] O. Peña, Y. Ma, M. Guilloux-Viry, and C. Moure, Appl. Surf. Sci. 254, 339 (2007), proceedings of the 13th International Conference on Solid Films and Surfaces.

[59] M. Pękala and V. Drozd, J. Alloys Comp. 437, 12 (2007).

[60] M. Hoffmann, V. S. Borisov, S. Ostanin, I. Mertig, W. Hergert, and A. Ernst, Phys. Rev. B 92, 094427 (2015).

[61] M. Hoffmann, V. Antonov, L. Bekenov, K. Kokko, W. J. Hergert, and A. Ernst, J. Phys.: Condens. Matter 30, 305801 (2018).

[62] P. Schiffer, A. P. Ramirez, W. Bao, and S.-W. Cheong, Phys. Rev. Lett. 75, 3336 (1995).

[63] A. Daoud-Aladine, J. Rodríguez-Carvajal, L. PinsardGaudart, M. T. Fernández-Díaz, and A. Revcolevschi, Phys. Rev. Lett. 89, 097205 (2002).

[64] D. J. García, K. Hallberg, C. D. Batista, M. Avignon, and B. Alascio, Phys. Rev. Lett. 85, 3720 (2000).

[65] M. H. Khan and S. Pal, Phys. Letters A 379, 401 (2015).

[66] C. Martin, A. Maignan, M. Hervieu, B. Raveau, Z. Jirák, M. M. Savosta, A. Kurbakov, V. Trounov, G. André, and F. Bourée, Phys. Rev. B 62, 6442 (2000).

[67] M. Geilhufe, S. Achilles, M. A. Köbis, M. Arnold, I. Mertig, W. Hergert, and A. Ernst, J. Phys.: Condens. Matter 27, 435202 (2015).

[68] R. D. Shannon, Acta Crystallogr. A 32, 751 (1976). 